\title{
Electrophoretic and Photolithographic Fluorescent Imaging with $t$-BOC-Protected Poly(t-BQzMA)-Polyaniline Conducting Particle System
}

\author{
Ki-Whan Chi, Ho Yun Hwang, Kwang Sun Rṿu, Jong-Man Kim, ${ }^{\dagger}$ Ung Chan Yoon, ${ }^{\ddagger}$ and Chan Woo Lee

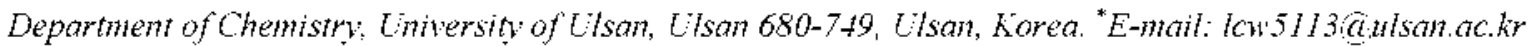 \\ Department of Chemical Engineering. Hanvang Lnvensin. Seoul 133-791, Korea \\ -Department of Chemistry Pusan National Lniversity, Busan 609-735, Korea \\ Received March 19, 2009, Accepted.April 30, 2009
}

Key Words: Electrophoretic. Photo-lithography. Fluorescence. Polyanuline. Quinizann

Among the conducting polymer. polyaniline-based photoimages are important since the conducting properties of polyaniline can be regulated by employing acid/base (doping/ dedoping) chemistry. ${ }^{1.2}$ Recently. we reported on noble constniction of electrophoretic fluorescence imaging prepared by nano-porous particles of polyaniline-fluorescein complex. ${ }^{3}$ These conducting particle system hold promise as fluorophores since they are partially polanzed and. as a result, they can be electrophoretically deposited onto a film of a mucro-patterned polyamline counter electrode.

In continung investigations aimed at the development of new systems for producing patterned fluorescence images. we explored the possibility of generating patterned images with conducting particles comprised of an acid-labile $t-\mathrm{BOC}$ group protected Qz-methacrylate poly mer ( $t$-BQzMA) and polyaniline. ${ }^{4.5}$ We hypothesized that protonation between the acidic phenol moiety of Qz polymer and a non-conducting poly aniline emeraldine base (EB) would not only render complexation but also the conductivity change of polyanuline by acid/base (doping/dedoping) chemistry. ${ }^{\text {l. }}$ It was assumed that the partially positive-charged polyaniline emeraldıne salt (ES) and negatively charged Qz polymers are separated and deposited on the counter electrode dunng the electrophoretic deposition process. and then patterned fluorescence images could be obtained. In order to demonstrate the feasibility of the above proposal. we developed a method to prepare micro-scale particles from the poly $(t-\mathrm{BQzMA})$-polyaniline system. A schematic of the procedure used for formation of the complex between

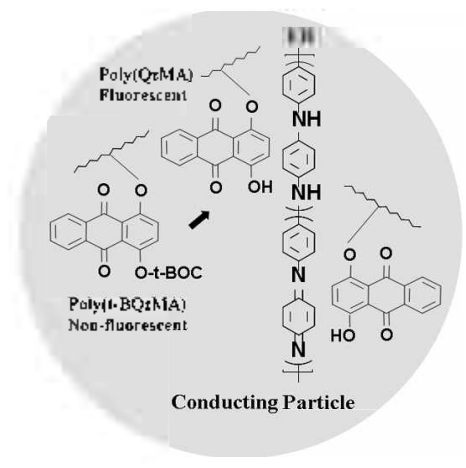

Figure 1. A schematic procedure for fonmation of the complex and conducting particle system incorporating poly $(t-B Q z M A)$ and polyaniline. the poly ( $t$-BQzMA) and polyaniline is shown in Fig. 1.

Polyaniline samples, poly $(t-\mathrm{BQzMA})$ and $t$-BOC-polyanuline were prepared as described in detal elsewhere. ${ }^{2,6,7}$ Simply, blending the polyaniline EB and the poly $(t-\mathrm{BQzMA})(1: 2$ by weight) in 1-methyl-2-pyrrolidinone (NMP) as solvent enabled the introduction of doped fluorescent labels on the porous particles as a consequence of proton transfer (or doping) under triphenylsulfonum triflate (TPSOTf. $5 \mathrm{wt} \%$ ) as a photo acid generator (PAG): the solution was irradiated with $250 \mathrm{~mm}$ UV for $30 \mathrm{~min}$, and then heated at $70^{\circ} \mathrm{C}$ for $1 \mathrm{~h}$ to give the deprotected phenol form from the protected Qz polymer. Since the polyamline in its EB state is basic, acidic phenol moiety of Qz polymers can exist in phenosy amon forms. Slow addition of NMP solution to ethanol followed by sonification leads to efficient formation of micro-scale particles. Scaming electron microscope (SEM) images. obtained following centrifugation and removal of large aggregates by filtration (l $\mu \mathrm{m})$. demonstrate that the nucro-sized particles are indeed generated by this method (see the experimental part) as shown in Fig. 2a. An ethanol solution. containing the particles obtained from the poly ( $t$-BQzMA) and the EB form of polyaniline. display a purple-pink (Fig. 2b) and bright yellow emission (Fig. 2c). The bright yellow enission indicates that they entit bright yellow fluorescence associated with the deprotected phenol form of poly (QzMA) and. as a result, they can be protonated to the EB form of polyaniline to give the ES form. In addition. the nucro-sized particles were found to be stable as ethanol suspensions without aggregation even after heating to reflux condition for $2+\mathrm{h}$. The particles are also stable in the presence of $5 \%$ tetrabutylanmonium fluoride salt. The observation of stable particles is significant in that most of conducting polymer particles probed to date tend to aggregate after prolonged storage. It is felt that the approach described above should be (a)

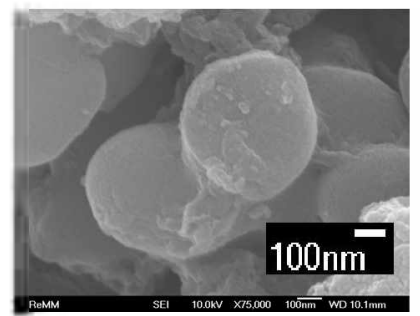

(b)

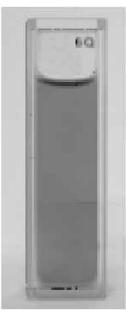

(c)

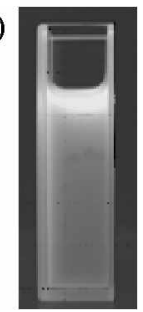

Figure 2. SEM (a), photographs of the conducting particles (ca. 0.5 $\mu \mathrm{m})$ without irradiation (b) and upon $365 \mathrm{~nm}$ irradiation (c). 
(a)

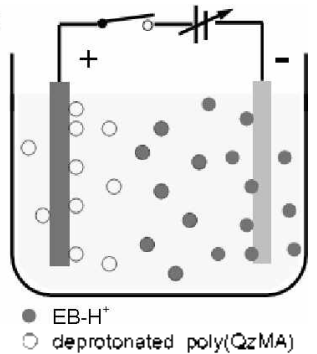

(b)

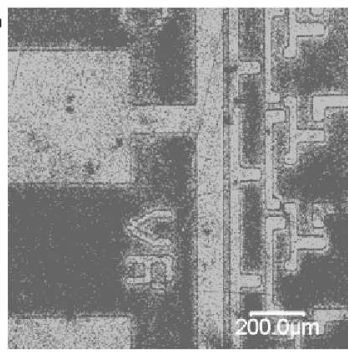

Figure 3. A schematic illustration of the device used for the electrophoretic micro-pattening with a solution containing conducting particles (a). The micro-pattemed image obtained after electrophoresis (b).

generally' applicable to the fabrication of stable conducting polymer blend system.

In most approaches for chemical patterning. irradiation is responsible for promoting irreversible modifications of photoresists in irreversible patterning processes. In contrast, $\mathrm{pH}$ sensitive or electro-sensitive materials which undergo reversible modification of their properties are highly desirable. Polyaniline is known to exist in various oxidation states. such as LB (leucoemeraldine base), $\mathrm{EB}$, and $\mathrm{PB}$ (pernigraniline base). Interconversions between these states permit the construction of electrochemically reversible redox cycle. Thus. we envisaged that reversible proton transfer could be constructed based on the control of the electrochemical redox cycle of polyaniline surface properties. Accordingly, the final phase of the current investigation focused on the electrophoretic patterning of the obtained polymer particles. A micro-patterned positive electrode (anode) was prepared according to the previously described method. ${ }^{3}$ Electrophoretic deposition on the micro-patterned polvaniline film with the polymer particles is illustrated schematically in Fig. 3a. When an electric field is applied the positively charged polyaniline are collect on the cathode. whereas the negatively charged Qz particles are deposited on the surface of the anode. Indeed. application of electric field to the solution of the particles leads to generation of patterned fluorescence images as displayed in Fig. $3 \mathrm{~b}$

To extend the photoimaging capability of the unique polyaniline-Qz polymer sy'stem. soluble polyaniline was synthesized by modifying with a $t$-BOC group: while unmodified polyaniline is not soluble in common organic solvents. The prepared $t$-BOC-polyaniline" is highly soluble in common solvents such as THF and chloroform. As a result of this solubility difference, conducting patterns can then be achieved in a photo-lithographic process by employing the Qz polymer. thereby capable of generating conducting and fluorescence patterns. To investigate the feasibility for potential application of microelectronic devices. thin films were prepared by spin-coating a THF solution containing poly $(t-\mathrm{BQzMA}), t$-BOC-polyaniline and PAG (TPSOTf. $5 \mathrm{w} \%$ ) on quartz substrates. The polymer film was irradiated with $250 \mathrm{~nm}$ UV for $1 \mathrm{~min}$ through a photomask in a contact mode followed by post-exposure-bake (PEB) at $120^{\circ} \mathrm{C}$ for $1 \mathrm{~min}$. As shown in Figure ta. after PEB treatment and $\mathrm{HCl}$ vapor exposure. complete deprotection of $t$-BOC groups in poly mer films was confirmed by UV spectral data. The unexposed film displays two absorption bands around $320 \mathrm{~nm}$ and $550 \mathrm{run}$. Upon exposure to UV light, the exciton
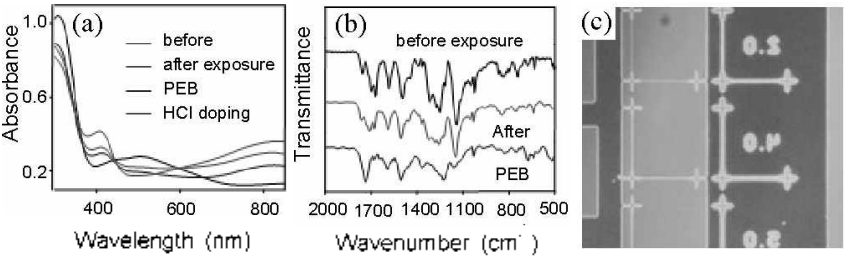

Figure 4. UV absorption spectral changes of a conducting polymer film containing $t$-BOC-polyaniline and poly $(t-\mathrm{BQzMA})$ on a quartz plate (a) and the corresponding IR spectral change. The micropattemed flurescent image obtamed by a photo-lithographic method $(\mathrm{c})$.

band around $580 \mathrm{~lm}$ decreases and two new bands appearing aroud 400 and $800 \mathrm{nu}$ slightly increase with increasing exposure dose. These bands are usually assigned to polaron bands originated from the conducting structure of polyaniline, i.e., doped. emeraldine salt. ${ }^{3}$ Further. visual inspection of the film showed color changes at the exposed areas. In addition, the deprotection of $t$-BOC groups in the polymer films were monitored by IR spectral changes (Figure 4b). During UV exposure and $\mathrm{PEB}$. the absorption bands at $1770 \mathrm{~cm}^{-1}$ associated with carbonyl of $t$-BOC groups gradually decreased with the PEB process. Simultaneously, the C-O bond (banding) intensity at $1120 \mathrm{~cm}^{-1}$ related to of $t$-BOC group decreased by removal of $t-\mathrm{BOC}$ group in the polymer. The deprotected polymer complex is insoluble in conmon organic solvents. and the un-irradiated part is soluble in THF solvent. Consequently. THF solution has been used as a developer to obtain good quality negative-tone patterns with fluorescence (Figure 4c). As expected. the fluorescence was effectively quenched when the fluorescent image patterns were treated with a $\mathrm{NaOH}$ solution since the base inhibits the intramolecular hydrogen bonding of the Qz moiety. Furthermore, fluorescence was recovered by acidic $\mathrm{HCl}$ treatment. These results imply that the fluorescence and conductivity property can be controlled by acid/base chemistry

In sunmary, application of an electric field to the solution containing the conducting particles enables formation of patterned fluorescence images by electrophoretic method. The results of electrophoretic fabrication and photo-lithographic imaging should stand as interesting and potential contributions to the fields of recording and sensing.

Acknowledgments. This work has been supported by the WCU research fund (R33-2008-000-10003) carried out in the Department of Chemistry. University of Ulsan.

\section{Refelences}

1. Chiang. I. C.: MacDiamid, A. G. Symth. Aet. 1986, 13, 193

2. MacDiamid, A. G. Sthth. Het. 2002, 125, 11 .

3. Chi, K.-W.; Hwang, H. Y.; Tin, S. H.; Yoon, G. S.; Teong, H. M: Kim, T.-M.; Lee, C. W. Chem Comm 2009, 13, 1647

4. Lee, C. W: Zhongzhe, Y,; Ahn, K. D.: Lee, S. H. Chem. Mater. 2002, 14,4572

5. Lee, C. W; Seo, Y. H.; Lee, S. H. Hacrontolecules $2004,37,4070$.

6. Lee, K. H.; Cho, S. U.; Park, S. H.; Heeger, A. J.; Lee, C. W.; Lee, S. H. Name 2006, 41,65 .

7. Lee, S. H.: Lee, D. H.: Lee, K. H.: Lee, C. W. Adv' Funt Mater. 2005, 15,1495 